\title{
COMPACTIFICATIONS OF HAUSDORFF SPACES
}

PETER A. LOEB ${ }^{1}$

1. Introduction. In this paper, we describe methods of imbedding a Hausdorff space $X$ in a compact space $\bar{X}$ so that each function in a given family of continuous functions on $X$ has a continuous extension to $\bar{X}$ and the family of extensions separates the points of $\bar{X}-X$. In particular, if $X$ is completely regular but not locally compact, then we shall exhibit a non-Hausdorff compactification which contains $X$ as an open subset and is bigger than the Stone-Čech compactification of $X$. (Of course, every compactification of $X$ is nonHausdorff if $X$ is not completely regular.) We shall also show that the completion of a metric space $M$ may be obtained as a subset of a compactification of $M$ by a rather simple construction.

By a compactification of a Hausdorff space $X$, we mean a compact space $\bar{X}$ which contains, as a dense subset, the image of $X$ under a fixed homeomorphism $f$. We usually do not distinguish between $X$ and $f(X)$, and we say that $\bar{X}$ contains $X$ as a dense subset. In what follows, $X$ is always a noncompact Hausdorff space, $\Delta \bar{X}$ denotes the closure of $\bar{X}-X$ in $\bar{X}$, and a mapping is always a continuous function. If $\bar{X}$ is Hausdorff, we say that $\bar{X}$ is a Hausdorff compactification of $X$. If $\bar{X}$ is not Hausdorff, however, we still assume that it satisfies the following properties:

I. Compact subsets of $X$ are closed in $\bar{X}$.

II. Any two distinct points $x$ and $y$ in $\Delta \bar{X}$ can be separated by disjoint open sets; i.e., there exist open sets $U$ and $V$ in $\bar{X}$ with $x \in U, y \in V$, and $U \cap V=\varnothing$.

III. For each point $x \in X$ there is at most one point $z \in \bar{X}-X$ such that $x$ and $z$ cannot be separated by disjoint open sets in $\bar{X}$.

Clearly, II and III are necessary conditions for the points in $\Delta \bar{X}$ to be separated by a family of continuous functions from $\bar{X}$ into a Hausdorff space; we shall show later that, together with Condition I, they are also sufficient. The following properties of $\bar{X}$ are consequences of $\mathrm{I}, \mathrm{II}$, and the fact that $X$ is dense in $\bar{X}$.

Presented to the Society, January 23, 1969; received by the editors July 5, 1968.

${ }^{1}$ This work was supported by National Science Foundation research grants GP5279 and GP-7537. 
Proposition 1.1. If $\bar{X}$ is a compactification of $X$, then: in $\bar{X}$

(i) Two distinct points in $X$ can be separated by disjoint open sets

(ii) The space $\bar{X}$ is $T_{1}$.

(iii) In $\bar{X}$, a sequence converges to at most one point.

(iv) If $X$ satisfies the first axiom of countabiiity then compact subsets of $\bar{X}$ are closed.

(v) If the compactification $\bar{X}$ satisfies the first axiom of countability, then $\bar{X}$ is Hausdorff.

(vi) $X$ is locally compact if and only if $X$ is open in $\bar{X}$ and $\bar{X}$ is Hausdorff.

Example. Following Arens [1], let $X$ be the set of all pairs of nonnegative integers such that each point other than $(0,0)$ is an open set and every neighborhood of $(0,0)$ contains all but a finite number of points in all but a finite number of columns $C_{n}$, where $C_{n}=\left\{(n, m): m \in Z^{+}\right\}$. Then $X$ is not first countable. Let $\bar{X}$ be the space $X$ together with a point $P$ whose neighborhoods omit at most a finite number of points in $X$. Any nonrepeating sequence with an infinite number of points in each column $C_{n}$ converges to $P$ and has cluster point $(0,0)$. Moreover, $\bar{X}-\{(0,0)\}$ is compact but not closed in $\bar{X}$.

Now let $\bar{X}$ and $\tilde{X}$ be two compactifications of $X$. By the notation $\bar{X} \geqq \tilde{X}$ we mean there is a mapping $T$ of $\bar{X}$ onto $\tilde{X}$ such that $T \mid X$ is the identity map. (To be more accurate, we should say that if $f$ and $g$ are the homeomorphisms of $X$ into $\bar{X}$ and $\tilde{X}$ respectively, then $T \circ f=g$.) If we also have $\tilde{X} \geqq \bar{X}$, then $T$ is a homeomorphism, and in this case we write $\bar{X} \cong \tilde{X}$.

2. $Q$-compactifications of $X$. Let $Q$ be a nonvoid family of continuous functions on $X$ with each $f \in Q$ having its range contained in a compact Hausdorff space $S_{f}$. Using the methods of [5], we now describe a compactification of $X$ which is the compactification defined in [5] when $X$ is locally compact.

Definition. Let $Y$ be the product space $\prod_{f \in Q} S_{f}$ and $e$ the evaluation map sending $X$ into $Y$. (For each $x \in X, e(x)(f)=f(x)$.) Set

$$
\Delta=\cap\{\overline{e(X-K)}: K \text { compact, } K \subset X\}
$$

and let $\bar{X}^{Q}$ be the (disjoint) union $X \cup \Delta$. Given an open set $U$ in $Y$ and a compact set $K \subset X$, we set $U_{K}=[U \cap \Delta] \cup\left[e^{-1}(U)-K\right]$. If $J$ is the topology on $\bar{X}^{Q}$ generated by the base consisting of all open 
sets in $X$ and all the sets $U_{K}$, then $\left(\bar{X}^{Q}, J\right)$ is called the $Q$-compactification of $X .^{2}$

It is not hard to show that $\bar{X}^{Q}$ is, indeed, a compactification of $X$; e.g., $\bar{X}^{Q}$ is compact since a net which is eventually in the complement of every compact subset of $X$ has a cluster point in $\Delta$. Clearly, $\bar{X}^{Q}$ also has the following properties:

THEOREM 2.1. Each function $f \in Q$ has a continuous extension mapping $\bar{X}^{Q}$ into $S_{f}$, and the family of these extensions separates the points in $\bar{X}^{Q}-X$. Moreover, $X$ is open in $\bar{X}^{Q}$. Thus, if $X$ is not locally compact, $\bar{X}^{Q}$ is neither Hausdorff nor a space which satisfies the first axiom of countability.

Next we show that these properties determine the compactification $\bar{X}^{Q}$ up to a homeomorphism.

THEOREM 2.2. Let $\tilde{X}$ be a compactification of $X$ such that each function $f$ in a nonvoid subfamily $Q_{0}$ of $Q$ has a continuous extension mapping $\tilde{X}$ into $S_{f}$ and these extensions separate the points in $\Delta \tilde{X}$. Then $\bar{X}^{Q} \geqq \tilde{X}$. If, moreover, $X$ is open in $\tilde{X}$ (e.g., if $X$ is locally compact) and if $Q_{0}=Q$, then $\bar{X}^{Q} \cong \tilde{X}$.

Proof. Let $\Gamma=\Delta \tilde{X}$ and recall that $\Delta=\bar{X}^{Q}-X$. Let $e, \bar{e}$, and $\tilde{e}$ be the evaluation maps sending $X, \bar{X}^{Q}$ and $\tilde{X}$ respectively into the product space $Y_{0}=\prod_{f \in Q_{0}} S_{f}$. Given $x_{0} \in \Delta$, let $N$ be a neighborhood of $\bar{e}\left(x_{0}\right)$ in $Y_{0}$, and let $\bar{N}$ be its closure in $Y_{0}$. Then $\tilde{e}^{-1}(\bar{N}) \cap \Gamma \neq \varnothing$, for otherwise $e^{-1}(\bar{N})=\tilde{e}^{-1}(\bar{N})$ is a compact subset of $X$, and $\bar{e}^{-1}(N)-e^{-1}(\bar{N})$ is a neighborhood of $x_{0}$ in $\bar{X}^{Q}$ that does not intersect $X$. Let $T\left(x_{0}\right)$ be the unique point in the intersection of all sets of the form $\tilde{e}^{-1}(\bar{N}) \cap \Gamma$ where $N$ ranges over the neighborhood system of $\bar{e}\left(x_{0}\right)$ in $Y_{0}$. One thus extends the identity mapping on $X$ to a function $T$ from $\bar{X}^{Q}$ into $\tilde{X}$, and in a similar way one shows that $T$ is onto. Clearly, $T$ is continuous at each point of $X$. Given $x_{0} \in \Delta$ and $U$ an open neighborhood of $T\left(x_{0}\right)$ in $\tilde{X}$, there is an open set $V$ in $Y_{0}$ such

? (Added July 19, 1968.) The author has learned of a manuscript, Minimum and maximum compactifications of arbitrary topological spaces, by R. F. Dickman, Jr., submitted in January 1967 to the Trans. Amer. Math. Soc. Using a different definition than the one given here and starting with an arbitrary topological space $X$ and a collection of mappings from $X$ into a single compact Hausdorff space $S$, Professor Dickman has proved the existence of a compactification $\alpha_{Q} X$ which has the properties established for $\bar{X}^{Q}$ by Theorems 2.1 and 2.2 below, and is thus equivalent to $\bar{X}^{Q}$. Professor Dickman has informed the author that these results will be included in a revised paper entitled Compactifications and real-compactifications of arbitrary topological spaces. 
that $\tilde{e}[\Gamma-U] \subset V$ and $\tilde{e}\left(T\left(x_{0}\right)\right)=\bar{e}\left(x_{0}\right) \notin \bar{V}$. Moreover, the set $K=\tilde{X}-\left[U \cup \tilde{e}^{-1}(V)\right]$ is a compact subset of $X$. Let $W=\bar{X}^{Q}$ $-\left[K \cup \bar{e}^{-1}(\bar{V})\right]$. Then $T(W) \subset U$, so $T$ is continuous at $x_{0}$, and thus $T$ is continuous on all of $\bar{X}^{Q}$. The rest of the proof is clear.

Corollary 2.3. If $Q_{0}$ is a nonvoid subset of $Q$, then $\bar{X}^{Q} \geqq \bar{X}^{Q_{0}}$.

Let $\bar{e}$ be the evaluation map sending $\bar{X}^{Q}$ into $Y$; then

$$
\bar{e}\left(\bar{X}^{Q}\right)=\overline{e(X \bar{X}},
$$

since $\bar{e}\left(\bar{X}^{Q}\right)=e(X) \cup \Delta$ in $Y$. One can, moreover, easily establish the following result:

Proposition 2.4. If there are no compact neighborhoods in $X$, then $\Delta$ is the closure of $e(X)$.

Examples. (1) If $Q$ consists of one mapping to a one point space, then $\bar{X}^{Q}$ is the Alexandroff one point compactification of $X$. (See $[4$, p. 150].)

(2) Let $X$ be the rational numbers in the real unit interval $[0,1]$, and let $Q$ consist of the single function $f(x)=x$. Then $\Delta$ is homeomorphic to $[0,1]$. A typical neighborhood of a point $y_{0} \in \Delta$ is given by a constant $\epsilon>0$ and a compact subset $K$ of $X$; it has the form

$$
\left\{y \in \Delta:\left|y-y_{0}\right|<\epsilon\right\} \cup\left\{x \in X-K:\left|x-y_{0}\right|<\epsilon\right\} .
$$

If $\tilde{X}=[0,1]$, then $\bar{X}^{Q} \geqq \tilde{X}$, but we do not have $\bar{X}^{Q} \cong \tilde{X}$.

(3) Let $H$ be an infinite-dimensional Hilbert space with the norm topology and let $H^{*}$ be its dual space with the weak topology. Let $X$ be the closed unit ball in $H, Q$ be the functions in $H^{*}$, and $e$ be the canonical map sending $H$ onto $H^{*}$. By Proposition 2.4, $\Delta=e(X)$, which is the closed unit ball with the weak topology. A typical neighborhood of a point $x \in \Delta$ has the form $[N \cap \Delta] \cup\left[e^{-1}(N) \cap X-K\right]$, where $N$ is a weak neighborhood of $x$ in $H^{*}$ and $K \subset X$ is compact in the norm topology.

Finally, we note that the results of this section can be applied to an arbitrary topological space $X$ if one works with closed and compact subsets of $X$ instead of compact subsets of $X$. The details are left to the reader.

3. Hausdorff $Q$-compactifications. In this section, we assume that $X$ is homeomorphic to its image $e(X)$ in the product space $Y=\prod_{f \in Q} S_{f}$ (see $[4$, p. 116]); $Q$ is the family of functions described in $\$ 2$. (If $X$ is locally compact, then, following Constantinescu and Cornea [3], one may adjoin all continuous real-valued functions 
with compact support to a given family of continuous functions to obtain a $Q$ which satisfies these assumptions.) Identify $X$ with $e(X)$; as is well known [7], [2], [4] the closure of $e(X)$ in $Y$ is a compact Hausdorff space which contains $X$ (i.e., $e(X)$ ) as a dense subset, and the functions in $Q$ have continuous extensions to the closure of $e(X)$. All the points in the closure of $e(X)$ are separated by these extensions. We call this closure the Hausdorff $Q$-compactification of $X$, and we denote it by $\bar{X}^{H Q}$. By Theorems 2.1 and 2.2, $\bar{X}^{Q} \geqq \bar{X}^{H Q}$, and $\bar{X}^{Q} \cong \bar{X}^{H Q}$ if and only if $X$ is locally compact. On the other hand, if there are no compact neighborhoods in $X$, then by Proposition $2.4, \Delta=\Delta \bar{X}^{Q}$ is homeomorphic to $\bar{X}^{H Q}$. The space $\bar{X}^{H Q}$ is unique in the following sense:

THEOREM 3.1. Let $X$ be a Hausdorff compactification of $X$ with each function in $Q$ having a continuous extension mapping $\tilde{X}$ into $S_{f}$. If these extensions separate the points of $\tilde{X}-X$, then $\bar{X}^{B Q} \cong \tilde{X}$.

Proof. We need only show that the evaluation map $\tilde{e}$ which sends $\tilde{X}$ onto $\bar{X}^{H Q}$ is injective. Assume that $\tilde{e}(x)=\tilde{e}(y)$ for some $x \in X$ and $y \in \tilde{X}-X$. Let $U \subset \tilde{X}$ be a neighborhood of $y$ such that $x \notin \bar{U}$, and let $C=\bar{U} \cap X$. Then $C$ is closed in $X$, so $\tilde{e}(C)=X \cap D$ where $D$ is closed in $\bar{X}^{H Q}$. Since $\tilde{e}(x)$ is not in $D, y$ is not in the closed set $\tilde{e}^{-1}(D)$. But this is impossible since $y$ is in the closure of $C=\tilde{e}^{-1}(D) \cap X$. Thus, $\tilde{e}$ is injective and therefore a homeomorphism.

Note that if $Q_{0}$ is a nonvoid subset of $Q$ and $X$ is homeomorphic to its image in $\prod_{f \in Q_{0}} S_{f}$, then since the projection of $\prod_{f \in Q} S_{f}$ onto $\prod_{f \in Q_{0}} S_{f}$ is continuous, $\bar{X}^{H Q} \geqq \bar{X}^{H Q_{0}}$.

EXAMPLES. (1) If $X$ is the set of rational numbers in $[0,1]$ and $Q$ consists of the single function $f(x)=x$, then $\bar{X}^{H Q}=[0,1]$. (Compare with Example 2 of $\$ 2$.)

(2) Let $X$ be a metric space with metric $d$, and let $Q$ be the family of functions $\left\{d_{x}: x \in X\right.$, where $d_{x}(y)=d(x, y)$ for all $\left.y \in X\right\}$. Each $d_{x}$ has its range in the interval $[0,+\infty]$. Set $X^{*}=\left\{z \in \bar{X}^{H Q}: \forall \epsilon>0\right.$ $\exists x \in X$ with $\left.d_{x}(z)<\epsilon\right\}$, and let $d^{*}(z, w)=\inf _{x \in X}\left[d_{x}(z)+d_{x}(w)\right]$ for each pair $(z, w)$ in $X^{*} \times X^{*}$. Then one can show that $d^{*}$ is a metric which generates the relative product topology on $X^{*}$ and $\left(X^{*}, d^{*}\right)$ is the completion of $(X, d)$.

(3) A similar construction gives the completion $X^{*}$ of a Hausdorff uniform space $X$ : If the uniform topology of $X$ is generated by the family of pseudometrics $\left\{d_{\alpha}: \alpha \in A\right\}$, and $Q=\left\{d_{\alpha}(x, \cdot): \alpha \in A, x \in X\right\}$, then

$$
X^{*}=\left\{z \in \bar{X}^{H Q}: \forall \epsilon>0 \text { and } \forall \alpha \in A, \exists x \in X \text { with } d_{\alpha}(x, z)<\epsilon\right\} .
$$


Using filters, Samuel [6] has constructed the largest compactification $X^{v}$ in which a given uniform space $X$ can be uniformly imbedded; the completion $X^{*}$ is the subset of $X^{v}$ consisting of all limits of Cauchy ultrafilters in $X$. However if $Q$ is any collection of uniformly continuous functions from $X$ into the real unit interval $I$ such that $\bar{X}^{H Q}$ exists, then $X$ is uniformly imbedded in $\bar{X}^{H Q}$. Moreover, any Hausdorff compactification $\tilde{X}$ in which $X$ is uniformly imbedded is of the form $\bar{X}^{H Q}$ where each $f \in Q$ maps $X$ uniformly into $I$. (See Theorem 4.2.) It follows that $\bar{X}^{v} \cong \bar{X}^{H \mathcal{u}}$ where $\mathcal{U}$ is the set of all uniformly continuous mappings of $X$ into $I$. Thus the compactifications used in the last two examples are, in general, smaller than $X^{v}$. If, for example, $X$ is the real line with 0 removed and $X$ has the additive uniform structure, then the compactification used in Example 2 is the one point compactification of the real line where as $X^{v}$ is "a space almost as complicated as the Cech compactification of the real line" $[6$, p. 124].

4. Properties of arbitrary compactifications. Let $\tilde{X}$ be any compactification of the Hausdorff space $X$ such that $\tilde{X}$ satisfies the three conditions in $\S 1$. Let $R \subset \tilde{X} \times \tilde{X}$ be the equivalence relation which consists of the diagonal set $\{(x, x): x \in \tilde{X}\}$ together with all pairs $(x, y)$ $\in \tilde{X} \times \tilde{X}$ for which there is a $z \in \tilde{X}-X$ such that neither $x$ nor $y$ can be separated from $z$ by disjoint open sets. As usual, $R[x]$ denotes the set of all points in $\tilde{X}$ equivalent to a point $x$, and for any set $A \subset \tilde{X}$, $R[A]=\bigcup_{x \in A} R[x]$.

Proposition 4.1. The relation $R$ has the following properties:

(i) For each $x \in \tilde{X}, R[x]$ is closed and therefore compact.

(ii) If $x$ and $y$ are points in $\tilde{X}$ with $R[x] \cap R[y]=\varnothing$, then there are disjoint open sets $U$ and $V$ in $\tilde{X}$ with $R[x] \subset U$ and $R[y] \subset V$.

(iii) If $z \in \tilde{X}-X$ and $U$ is an open neighborhood of $z$, then $R[z]$ is contained in the closure $\bar{U}$ of $U$.

(iv) If $x \in X \cap \Delta \tilde{X}$, then $R[x]=\{x\}$.

(v) If $C$ is compact in $\tilde{X}$, then $R[C]$ is closed.

Proof. We shall only prove $(\mathrm{v})$. We show first that $R[C] \cap \Delta \tilde{X}$ is closed. If $\left\{z_{\alpha}\right\}_{\alpha \in A}$ is a net in $R[C] \cap \Delta \tilde{X}$ and $\left\{z_{\alpha}\right\}$ converges to $z \in \Delta \tilde{X}$, then for each $\alpha$ in the index set $A$ there is a point $x_{\alpha}$ in $R\left[z_{\alpha}\right] \cap C$. Let $x \in C$ be a cluster point of the net $\left\{x_{\alpha}\right\}_{\alpha \in A}$. Given open neighborhoods $U$ and $V$ of $z$ and $x$ respectively, there is an $\alpha \in A$ such that $z_{\alpha} \in U$ and $x_{\alpha} \in V$. Since $x_{\alpha} \in R\left[z_{\alpha}\right]$ and either $x_{\alpha}=z_{\alpha}$ or $z_{\alpha} \in \tilde{X}-X$, it follows that $U \cap V \neq \varnothing$, and thus $z \in R[C]$. We have shown that $R[C] \cap \Delta \tilde{X}$ is closed. 
Assume now that $y_{0} \notin R[C]$. Then for each set $R[x] \subset R[C]$, there is a pair of disjoint open sets $U$ and $V$ in $\tilde{X}$ with $R[x] \subset U$ and $y_{0} \in V$. Thus the compact set $C \cup[R[C] \cap \Delta \tilde{X}]$ is contained in a finite union of open sets $\left\{U_{i}: i=1,2, \cdots, n\right\}$ such that $y_{0} \notin \cup_{i=1}^{n} \bar{U}_{i}$. But by (iii), $R[C]$ is contained in $\bigcup_{i=1}^{n} \bar{U}_{i}$. Thus $R[C]$ is closed.

We next show that $\tilde{X} / R$ is Hausdorff; clearly, $R$ is the finest relation for which this can be true. It follows that the arbitrarily chosen compactification $\tilde{X}$ is comparable with an appropriate $Q$-compactification. The following theorem for the case that $\tilde{X}$ is Hausdorff is due to Čech [2].

THEOREM 4.2. Let $\widetilde{Q}$ be the set of all mappings of $\tilde{X}$ into the unit interval $[0,1]$, and let $Q$ be the set of restrictions $\{f \mid X: f \in \tilde{Q}\}$. Then $\widetilde{Q}$ separates the points in $\Delta \tilde{X}$, and thus $\bar{X}^{Q} \geqq \tilde{X}$. If $X$ is open in $\tilde{X}$, then $\bar{X}^{Q} \cong \tilde{X}$. If $\tilde{X}$ is Hausdorff, then $\bar{X}^{H Q} \cong \tilde{X}$.

Proof. By Theorems 2.2 and 3.1, we need only show that $\widetilde{Q}$ separates the points in $\Delta \tilde{X}$. Let $P$ be the projection of $\tilde{X}$ onto the quotient space $\tilde{X} / R$. If $P(x)$ and $P(y)$ are distinct points in $\tilde{X} / R$, then there are disjoint neighborhoods $U$ and $V$ of $R[x]$ and $R[y]$ in $\tilde{X}$. Let $C=X-U$ and $D=X-V$. Then $R[C]$ is a closed set with $R[C] \cap R[x]$ $=\varnothing ; R[D]$ is a closed set with $R[D] \cap R[y]=\varnothing$, and $R[D] \cup R[C]$ $=\tilde{X}$. Therefore, $P(R[C])$ and $P(R[D])$ are closed sets in $\tilde{X} / R$ with $P(x) \notin P(R[C]), \quad P(y) \notin P(R[D])$, and $P(R[C]) \cup P(R[D])=\tilde{X} / R$. Thus $\tilde{X} / R$ is Hausdorff, and the theorem follows from Urysohn's lemma.

CoROllaRY 4.3. Every compactification of a locally compact Hausdorff space is a Q-compactification. Every Hausdorff compactification of a completely regular space is a Hausdorff Q-compactification.

Finally, we let $g$ be the set of all mappings of $X$ into the unit interval $[0,1]$, and we consider the compactifications $\bar{X}^{s}$ and $\bar{X}^{H s}$. Of course, $\bar{X}^{H s}$ is only defined if $X$ is completely regular, and it is the Stone-Cech compactification of $X$.

THEOREM 4.4. Let $\tilde{X}$ be any compactification of $X$. Then $\bar{X}^{s} \geqq \tilde{X}$, and as is well known, $\bar{X}^{H s} \geqq \tilde{X}$ if $\tilde{X}$ is Hausdorff.

Proof. The result follows from Theorem 4.2, Corollary 2.3 and the remark following Theorem 3.1.

We have shown that if $X$ is completely regular, then $\bar{X}^{s} \geqq \bar{X}^{H s}$, while $\bar{X}^{s} \cong \bar{X}^{H s}$ only if $X$ is locally compact. If $X$ is not locally compact, then $\bar{X}^{\sharp}$ dominates a larger class of compactifications than the Stone-Cech compactification $\bar{X}^{H s}$. Indeed if $X$ is not completely 
regular, $\bar{X}^{H s}$ is not even defined. Moreover, $X$ is always an open subset of $\bar{X}^{s}$, but only when $X$ is locally compact is it open in $\bar{X}^{H s}$.

\section{REFERENCES}

1. R. Arens, Note on convergence in topology, Math. Mag. 23 (1950), 229-234.

2. E. Čech, On bicompact spaces, Ann. of Math. 38 (1937), 823-844.

3. C. Constantinescu and A. Cornea, Ideale Ränder Riemannscher Flächen, Ergebnisse der Mathematik und ihrer Grenzgebiete, Bd. 32, Springer-Verlag, Berlin, 1963.

4. J. L. Kelley, General topology, Van Nostrand, Princeton, N. J., 1955.

5. P. A. Loeb, A minimal compactification for extending continuous functions, Proc. Amer. Math. Soc. 18 (1967), 282-283.

6. P. Samuel, Ultrafilters and compactification of uniform spaces, Trans. Amer. Math. Soc. 64 (1948), 100-132.

7. A. Tychonoff, Über die topologische Erweiterung von Räumen, Math. Ann. 102 (1929), 544-561.

University of California, Los Angeles and

UNIVERSITY OF ILLINOIS, URBANA 